\title{
Differential Cross-Polarized Wireless Communications
}

\author{
Siamack Ghadimi \\ Isfahan, Iran \\ Email: siamack.ghadimi@gmail.com
}

How to cite this paper: Ghadimi, S. (2019) Differential Cross-Polarized Wireless Communications. Wireless Engineering and Technology, 10, 34-40.

https://doi.org/10.4236/wet.2019.102003

Received: March 20, 2019

Accepted: April 25, 2019

Published: April 28, 2019

Copyright $\odot 2019$ by author(s) and Scientific Research Publishing Inc. This work is licensed under the Creative Commons Attribution International License (CC BY 4.0).

http://creativecommons.org/licenses/by/4.0/

\begin{abstract}
This paper presents a novel method for polarized antenna transmission utilizing a differential technique. The method uses a linear cross-polarized antenna, for example, a vertical and horizontal polarized antenna, improving performance and power efficiency of wireless communications. Hence a linear relationship between the capacity of the systems and the number of cross-polarized antennas exists. The result shows the fact that this method enhances the SNR comparing to other systems. This solution is simple, compact and does not require any bandwidth expansion.
\end{abstract}

\section{Keywords}

Cross-Polarized Antennas, Differential Communication, SISO, MIMO

\section{Introduction}

The next generation of wireless communications demands a high data rate to meet requirements from various applications. There are several different ways to improve communication performance, but these ways are not cheap, and/or simple. For example, antenna diversity requires a physical space of the order of a wavelength between antennas [1], which in the case of a compact device is not applicable, wherein a cross-polarized antenna will give a compact solution. Transmit diversity is an effective way to improve fading, but is not sufficient.

Polarization is one of the key features for tomorrow's transmission systems. As we know, horizontal, vertical, left- and right-hand circular polarized transmissions will not interfere with each other, as they are differently polarized. This means that signals via the same carrier frequency can transmit and be received simultaneously on orthogonally polarized waves, without any requirement of spatial separation [2]. 
Note: The antennas polarization is distinguished as Co-polarized or Spatial ( || ), Dual-polarized ( |-) or Cross-polarized (+) [3].

A cross-polarized antenna is an integration of two co-located antennas with orthogonal polarization. To convey the idea of a channel model for a cross-polarized transceiver antenna, the paper assumes that the channel is frequency-flat. Then the signal model is given by:

$$
r=\sqrt{E} \cdot H_{t} \cdot s+n
$$

where the $\boldsymbol{r}$ is the received signal vector, the $\sqrt{E}$ is the average energy at each transmitter antenna, for every symbol average. The $\boldsymbol{H}_{t}$ is the total channel polarization matrix. The $s$ is the signal vector with normalized average energy, and the $\boldsymbol{n}$ is noise vector with i.i.d. zero-mean complex Gaussian. The dimension of $s, \boldsymbol{H}_{t}$ and $\boldsymbol{n}$ depends on the number of transmitter polarized antennas.

The channel polarization matrix $\boldsymbol{H}_{t}$, represents co and cross-polarized components, cross-correlation and cross-coupling of energy from one polarization mode (vertical/horizontal $0^{\circ} / 90^{\circ}$ or slanted $\pm 45^{\circ}$ ) to the other polarization mode.

The channel polarization matrix $\boldsymbol{H}_{t}$ decomposed into the sum of the fixed (line of sight) part and the average random (fast fading) part.

$$
H_{t}=\underbrace{\sqrt{\frac{K}{1+K}} \bar{H}_{n \times m}}_{\text {Fixed part }}+\underbrace{\sqrt{\frac{1}{1+K}} \tilde{H}_{n \times m}}_{\text {Variable part }}
$$

where $K$ is the Rician $K$-factor and $\overline{\boldsymbol{H}}_{n \times m}$ is a deterministic or time-static channel matrix, with a received contribution from Line of Sight (LOS), and $\tilde{\boldsymbol{H}}_{n \times m}$ is a random or time-variant channel matrix with a received contribution from Non Line of Sight (NLOS) for an $n$-receiver $m$-transmitter system. Hence if $K=0$ then $\boldsymbol{H}_{t}$ will represent a Rayleigh fading channel and if $K \rightarrow \infty$ it will represent a static channel [4] [5].

Now if we are assuming that the transmitter and receiver antenna consist of $n$ pairs and $m$ pairs of cross-polarized, vertical $(\mathrm{V})$ and horizontal $(\mathrm{H})$, antennas respectively, the channel matrix for LOS and NLOS component can be written as:

$$
\begin{aligned}
\bar{H}_{L O S} & =\left[\begin{array}{ll}
\bar{H}_{n \times m}^{V V} & \bar{H}_{n \times m}^{V H} \cdot A_{z} \\
\bar{H}_{n \times m}^{H V} & \bar{H}_{n \times m}^{H H} \cdot A_{z}
\end{array}\right] \\
\tilde{H}_{N L O S} & =\left[\begin{array}{ll}
\tilde{H}_{n \times m}^{V V} & \tilde{H}_{n \times m}^{V H} \cdot A_{z} \\
\tilde{H}_{n \times m}^{H V} & \tilde{H}_{n \times m}^{H H} \cdot A_{z}
\end{array}\right]
\end{aligned}
$$

where $\boldsymbol{H}^{V V}$ and $\boldsymbol{H}^{H H}$ represent co-polarized components while $\boldsymbol{H}^{H V}$ and $\boldsymbol{H}^{V H}$ represent cross-polarized components.

For LOS the elements of matrix $\overline{\boldsymbol{H}}_{n \times m}^{x y}(x, y=V, H)$ can be obtained according to Malu's law:

$$
\bar{h}=\frac{\mathrm{e}^{-j k d_{1}}}{d_{1}} G_{R} G_{T} \cdot \cos \theta_{r}
$$


where $\cos \theta_{r}$ is the polarization mismatch component and $\theta_{r}$ is the polarization mismatch rotation angle between the transmitter and receiver. If the transmitter and receiver are all strictly aligned, namely co-polarized, then $\theta_{r}$ is equal to 0 for the channel between $V V$ and $H H$, while $\theta_{r}$ is equal to $\pi / 2$ for the channel between $H V$ and $V H$, namely orthogonally oriented. But in reality the transmitter and receiver antenna are not exactly aligned, therefore if the polarization mismatch component for $V V$ and $H H$ channel is $\cos \theta_{r}$ then for $V H$ and $H V$ the polarization mismatch becomes $\cos \left(\pi / 2-\theta_{r}\right)$. Thus the channel components for LOS are:

$$
\begin{gathered}
\bar{h}^{x, x}=\frac{\mathrm{e}^{-j k d_{1}}}{d_{1}} G_{R}^{x} G_{T}^{x} \cdot \cos \theta_{r}, \quad x=V, H \\
\bar{h}^{x, y}=\frac{\mathrm{e}^{-j k d_{1}}}{d_{1}} G_{R}^{x} G_{T}^{y} \cdot \cos \left(\pi / 2-\theta_{r}\right), \quad x, y=V, H
\end{gathered}
$$

The $A_{z}$ in the Equations (3) and (4) is the azimuthal displacement of the transmitter and receiver antennas, for horizontally polarized transmitting signals, so if we assume that the angle of arrival at the receiver is $\psi^{A}$ and the angle of departure at the transmitter is $\psi^{D}$, then $A_{z}=\cos \psi^{A} \cdot \cos \psi^{D}$.

For NLOS, the element of matrix $\tilde{\boldsymbol{H}}_{n \times m}^{x y}(x, y=V, H)$ can be obtained according to Snell's law:

$$
\begin{gathered}
\tilde{h}^{x, x}=\sum_{i=1}^{n s} \frac{\mathrm{e}^{-j k d_{2, i}}}{d_{2, i}} G_{R, i}^{x} G_{T, i}^{x} \cdot \alpha_{i}\left(\phi_{i}\right) \cdot \cos \theta_{r, i}, \\
x=V, H \\
\tilde{h}^{x, y}=\sum_{i=1}^{n s} \frac{\mathrm{e}^{-j k d_{2, i}}}{d_{2, i}} G_{R, i}^{x} G_{T, i}^{y} \cdot \alpha_{i}\left(\phi_{i}\right) \cdot \cos \left(\pi / 2-\theta_{r, i}\right), \\
x, y=V, H \\
\Rightarrow \lim _{n s \rightarrow \infty} \simeq \sqrt{P_{r}^{N L O S}}\left[\begin{array}{ll}
h_{v v} & h_{v h} \\
h_{h v} & h_{h h}
\end{array}\right]
\end{gathered}
$$

where $\alpha_{i}\left(\phi_{i}\right)$ is the reflection components, which describe the total amplitude and phase changes due to the reflection, diffraction and scattering on propagation paths. The $n s$ is the number of paths. In other words, $\tilde{\boldsymbol{H}}_{N L O S}$ is the summation of all multipath field components for transmitter and receiver antenna pairs, which according to the central limit theorem can be approximated as i.i.d. Gaussian model. When $n s$ is big enough, the channel may be approximated by the Rayleigh channel, where $P_{r}^{N L O S}$ is the total received power, $h_{v v}, h_{h h}, h_{v h}$ and $h_{h v}$ are i.i.d. complex Gaussian random variables.

The idea behind this differential cross-polarized technique is based on the cross-polarized transceiver. To this end, consider a wireless transceiver system, each one with a linear cross-polarized antenna, e.g. vertical (V) and horizontal $(\mathrm{H})$ polarized antennas, as Figure 1 shows.

Just to be clear, the transmitter and receiver deal with one physical cross-polarized 
antenna each, but the channel is based on 2 input and 2 output channel. Consequently, each polarization mode behaves as an independent physical channel.

Now let's, for simplicity, consider only the LOS signals shown in Figure 2.

Assume signal $s$ is transmitted by the vertical polarized antenna, and the complement of this signal, namely $\overline{\boldsymbol{s}}$, is transmitted simultaneously by the horizontal polarized antenna over a noisy channel. The channel noise will be added to both signals.

Now if received signal $\tilde{\overline{\boldsymbol{S}}}$ by horizontal polarized antenna is $180^{\circ}$ phase shifted and added to received signal $\tilde{\boldsymbol{s}}$ by the vertical polarized antenna, the noises will cancel each other.

But in reality, because of the reflection, scattering, depolarization, phase errors, and symmetric leakage from one polarization mode $V / H$ to another polarization mode $V / H$ (Figure 3), although the system will not completely cancel out the noise as Figure 2 shows. However, it will significantly improve the performance of the system and decrease BER, as the simulation result will show in the next section.

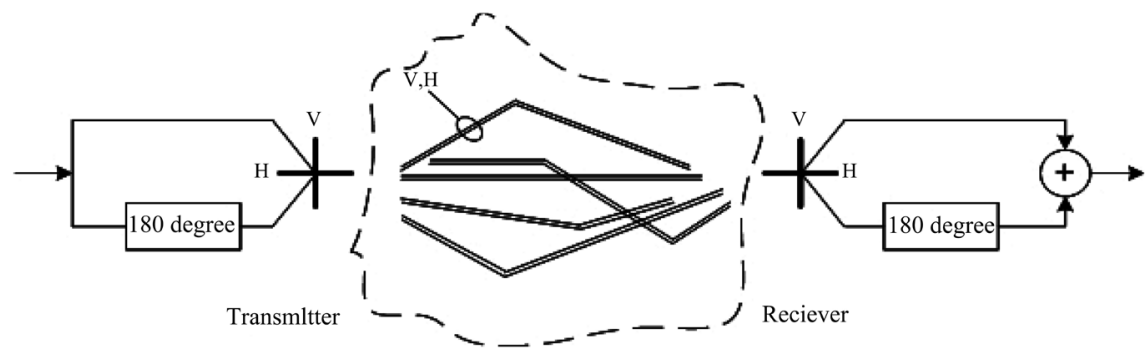

Figure 1. Polarized transceiver.

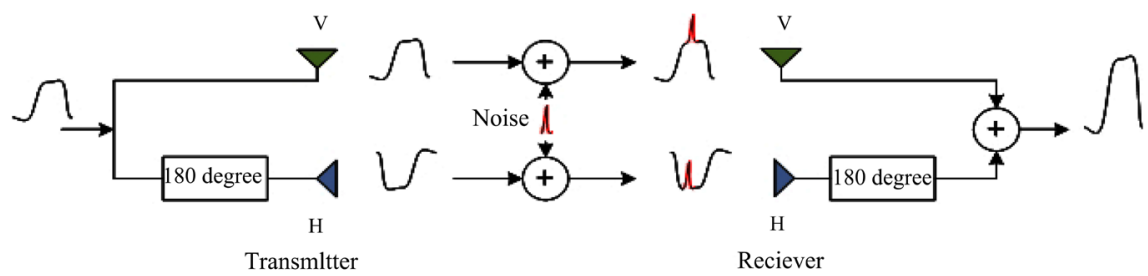

Figure 2. Polarized transceiver in a noisy-channel.

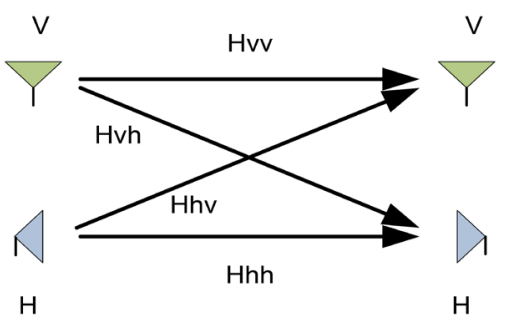

Transmitter

Cross-polarized

Reciever

Figure 3. Cross-polarized system. 


\section{Simulation and Result}

Simulation was done in MATLAB for computing the BER with BPSK modulation over an AWGN channel.

If we assume signal $s$ is transmitted by the horizontal antennas and simultaneously it's complement $\overline{\boldsymbol{s}}$ by the vertical antennas, then the received signals at the receiver, from the horizontal $\tilde{\boldsymbol{s}}$ and vertical $\tilde{\overline{\boldsymbol{s}}}$ antennas are:

$$
\begin{gathered}
\tilde{s}=\left(s \cdot h_{L O S}^{H H}+s \cdot h_{L O S}^{V V}\right)+\left(\bar{s} \cdot h_{L O S}^{H V}+\bar{s} \cdot g_{L O S}^{V H}\right) \\
\tilde{\bar{s}}=\left(s \cdot h_{N L O S}^{H H}+s \cdot h_{N L O S}^{V V}\right)+\left(\bar{s} \cdot h_{N L O S}^{H V}+\bar{s} \cdot g_{N L O S}^{V H}\right)
\end{gathered}
$$

where $h_{\chi}^{H H}, h_{\chi}^{V V}, h_{\chi}^{H V}$ and $h_{\chi}^{V H}, \quad \chi=\{L O S, N L O S\}$ are the channel coefficients for transmission between transmitter and receiver horizontal-horizontal, vertical-vertical, horizontal-vertical, and vertical-horizontal antennas respectively.

Finally by phase shifting $180^{\circ}$ and adding these two signals together, the received signal is:

$$
r=(\tilde{s}+n)+\overline{(\tilde{\bar{s}}+n)}
$$

For simulation, the polarization rotation angle between the transmitter and receiver antennas and the azimuthal displacement angle are assumed $30^{\circ}$. For a fair comparison, Rician K-factor was chosen as 10, for a Rician fading channel as well as for a Rayleigh fading channel.

It is also assumed that the total transmit power is the same for all simulation scenarios to allow a fair comparison, and the receiver has perfect knowledge of the channel.

Figure 4 depicts the Differential Cross-Polarized Technique vs. Single-input single-output (SISO) system and vs. Multiple-input and multiple-output (MIMO) $2 \times 2$ antennas and vs. MIMO dual-polarized $2 \times 2$ antennas. As shown in the Figure 4, the performance of the Differential Cross-Polarized Technique is superior.

This result shows that the Differential Cross-Polarized Technique, when comparing with other systems, not only performs better, but provides a more robust and efficient transmission, and enhances the SNR of the received signal by around $3 \mathrm{~dB}$. This is particularly true when the antennas are fixed and the rotation angle between the receiver and transmitter antenna is small (except for movement of scatters around the system, where there is a very low polarization mismatch for the cross-polarized antenna system).

Figure 5 and Figure 6 portray the cross-polar discrimination (XPD) for different $\mathrm{Rx} / \mathrm{Tx}$ rotation angles, when $\mathrm{SNR}=10 \mathrm{~dB}, \mathrm{~K}$-factor $=10$, and the azimuthal displacement angle are assumed $30^{\circ}$. Figure 5 shown that, the XPDV channel is slightly larger than the XPDH channel, because of the azimuthal rotation angle. Note that in Figure 6 when the rotation angle is about $40^{\circ}$ the XPDH is 1 , while the XPDV for $55^{\circ}$ is 1 . The reason is the effect of azimuthal on $H H$ channel, which make the XPDV higher than the XPDH. 


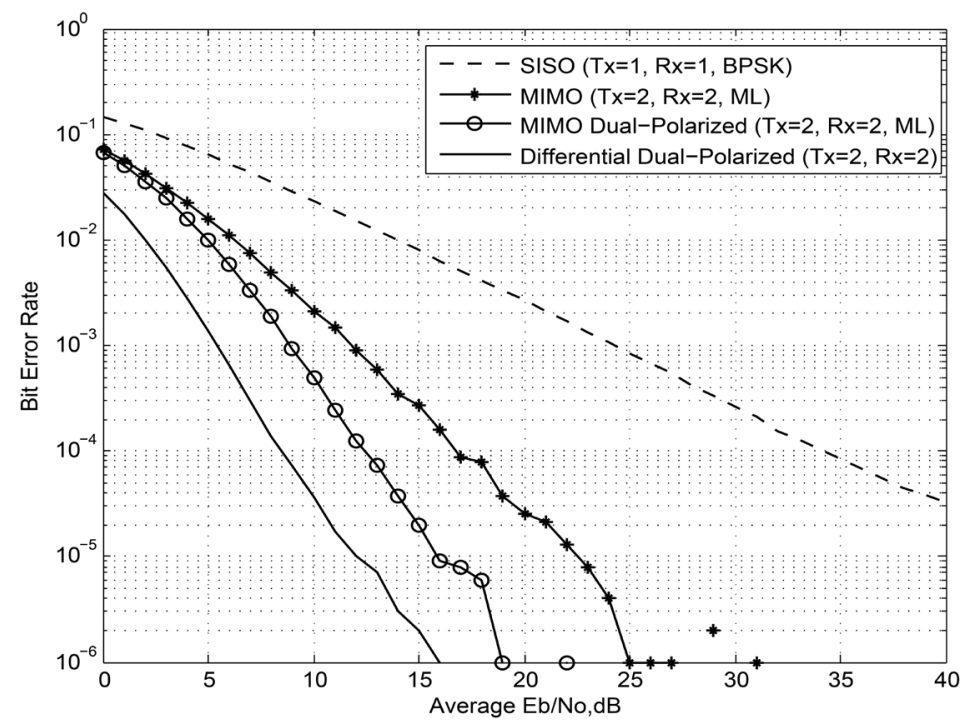

Figure 4. Simulation of BER for differential cross-polarized technique.

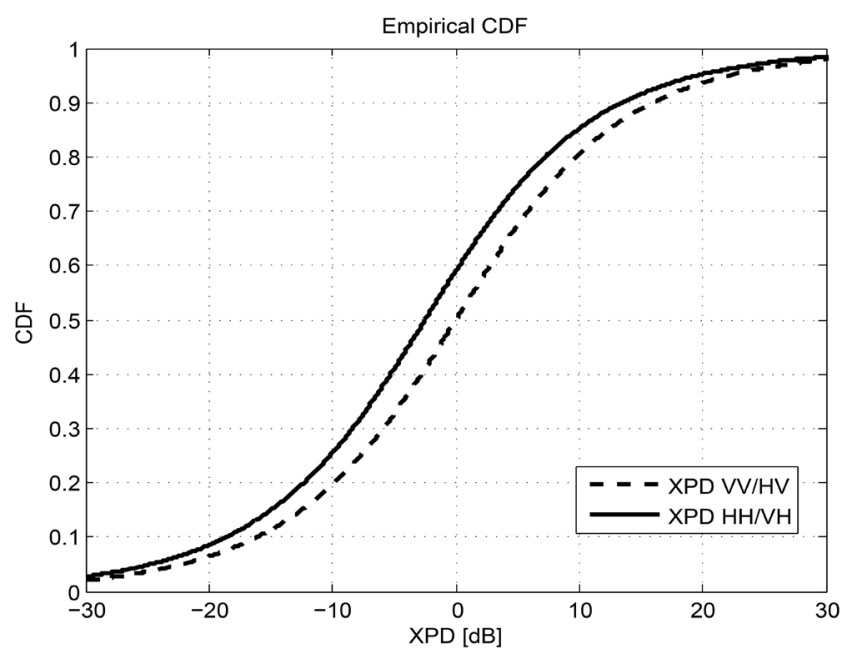

Figure 5. XPD function of antenna rotation angle.

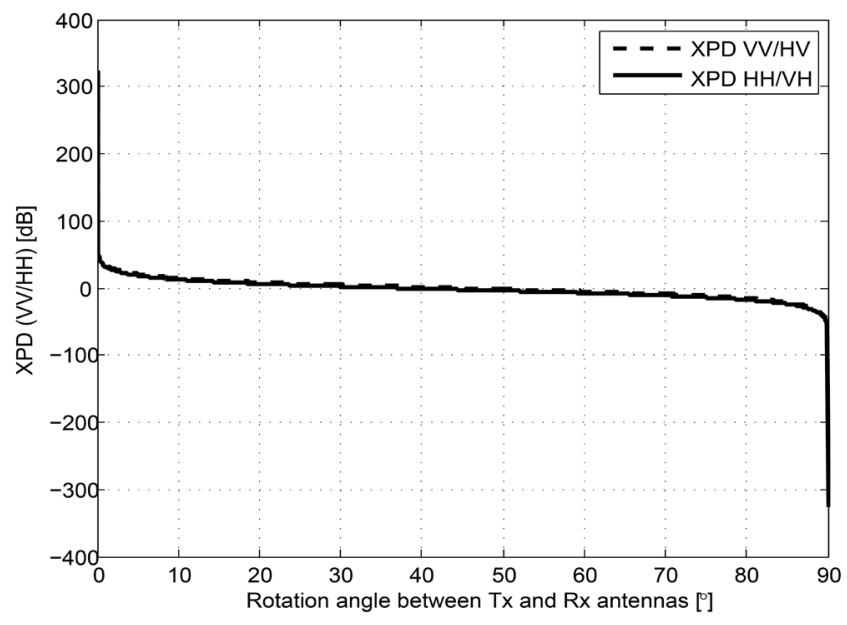

Figure 6. CDF function of XPD. 


\section{Conclusions}

In this paper, a new technique for wireless communication systems is suggested based on differential technique.

The proposed system "Differential Cross-Polarized Wireless Communications" improves the performance, power efficiency, and link reliability, with a minimal increase in complexity.

The other advantage of the Differential Cross-Polarized Technique is that it is both a cost- and space-effective alternative, where two spatially separated co-polarized antennas are replaced by a single antenna with orthogonal polarization.

The proposed system imposed, simulated and compared favourably with an existing SISO system and MIMO $2 \times 2$ antennas and MIMO dual-polarized $2 \times 2$ antennas in a fading channel.

Further research can be initiated to analyze the system in the effect of movement in a noisy environment.

Finally, the most complicated solution is the simplest one.

\section{Conflicts of Interest}

The author declares no conflicts of interest regarding the publication of this paper.

\section{References}

[1] Jiang, J.-S. and Ingram, M.A. (2005) Spherical-Wave Model for Short-Range Mimo. IEEE Transactions on Communications, 53, 1534-1541.

https://doi.org/10.1109/TCOMM.2005.852842

[2] Ghadimi, S. (2013) Performance Assessment of Wireless Two Way Relay Channel Systems. Ph.D. Dissertation, Univ. of Western, London Ontario. http://ir.lib.uwo.ca/etd/1845/

[3] Balanis, C.A. (2005) Antenna Theory: Analysis and Design. 3rd Edition, Wiley, Hoboken.

[4] Bolcskei, H., Nabar, R.U., Erceg, V., Gesbert, D. and Paulraj, A.J. (2001) Performance of Spatial Multiplexing in the Presence of Polarization Diversity. 2001 IEEE International Conference on Acoustics, Speech, and Signal Processing,), Salt Lake City, UT, 7-11 May 2001, 2437-2440. https://doi.org/10.1109/ICASSP.2001.940493

[5] Oestges, C., Erceg, V. and Paulraj, A.J. (2004) Propagation Modeling of Mimo Multipolarized Fixed Wireless Channels. IEEE Transactions on Vehicular Technology, 53, 644-654. https://doi.org/10.1109/TVT.2004.827149 